\title{
Propriedades reológicas de sucos mistos de manga, goiaba e acerola adicionados de fitoquímicos
}

Rheological properties of mixed juices of mango, guava and acerola with added phytochemicals

\section{Autores | Authors}

$\bowtie$ Aurélia Santos FARAONI

Universidade Federal de Viçosa (UFV) Departamento de Tecnologia de Alimentos (DTA) CEP: 36570-000 Viçosa/MG - Brasil e-mail: faraoniaurelia@yahoo.com.br

Afonso Mota RAMOS Danilo Bergamaschi GUEDES Marcos Roberto Moacir Ribeiro PINTO

Universidade Federal de Viçosa (UFV) Departamento de Tecnologia de Alimentos (DTA) Viçosa/MG - Brasil e-mail:amramos@ufv.br danilobguedes@gmail.com marcosrmrp@gmail.com

$\triangle$ Autor Correspondente / Corresponding Author

Recebido / Received: 30/05/2011 Aprovado / Approved: 24/09/2012 Publicado / Published: mar./2013

\section{Resumo}

O objetivo deste trabalho foi estudar o comportamento reológico dos sucos mistos de manga, goiaba e acerola, adicionados de fitoquímicos, em sete temperaturas $\left(10,20,30,40,50,60\right.$ e $\left.70^{\circ} \mathrm{C}\right)$, e o efeito da temperatura na viscosidade aparente. As análises reológicas foram conduzidas em um reômetro de cilindros concêntricos, marca Brookfield, modelo R/S plus SST 2000. Os valores experimentais de tensão de cisalhamento versus taxa de deformação foram ajustados pelos modelos de Ostwald-de-Waele (Lei da Potência), Casson e Herschel-Bulkley. Observou-se que os três modelos apresentaram altos coeficientes de determinação $\left(R^{2} \geq 0,978\right)$, indicando que qualquer um destes pode ser utilizado para descrever o comportamento reológico dos sucos, caracterizados como fluidos não newtonianos com características pseudoplásticas. O efeito da temperatura no comportamento reológico dos sucos foi descrito pela Equação de Arrhenius e foi possível observar um decréscimo no valor da viscosidade aparente com o aumento da temperatura.

Palavras-chave: Sucos de frutas; Comportamento reológico; Viscosidade; Efeito da temperatura.

\section{Summary}

The aim of this work was to study the rheological behaviour of mixed juices of mango, guava and acerola with added phytochemicals at seven temperatures $\left(10,20,30,40,50,60\right.$ and $\left.70{ }^{\circ} \mathrm{C}\right)$, and the effect of temperature on the apparent viscosity. The rheological analyses were carried out using a Brookfield R/S plus SST 2000 rheometer with concentric cylinders. The experimental data for shear stress versus shear rate were fitted to the following models: Ostwald-de-Waele (Power Law), Casson and Herschel-Bulkley. The three models presented high determination coefficient values $\left(R^{2} \geq 0.978\right)$, indicating that any of them could be used to describe the rheological behaviour of the juices. The juices presented non-Newtonian behaviour and pseudoplastic characteristics. The effect of temperature on the rheological behaviour of the juices was described by the Arrhenius equation. The values for apparent viscosity decreased with increase in temperature.

Key words: Fruit juices; Rheological behaviour; Viscosity; Effect of temperature. 


\section{Introdução}

A preocupação das pessoas com a saúde tem elevado o consumo de frutas e de seus derivados nos últimos anos, pois, cada vez mais, estudos têm demonstrado que o consumo regular de frutas é um grande aliado na redução do risco de desenvolvimento de diversas doenças, uma vez que são ricas em vitaminas, carotenoides, compostos fenólicos e outros componentes importantes.

Os fitoquímicos (do grego, fitos = planta) poderiam ser definidos, simplesmente, como compostos químicos presentes ou provenientes das plantas. Sua definição é, usualmente, complementada pelas atribuições especiais desses compostos; não basta, portanto, vir do reino vegetal, é necessário que o composto promova efeitos benéficos ao organismo, para que possa ser considerado um fitoquímico (GOMES, 2007). Entre os mais importantes, estão os terpenoides, que incluem carotenoides, fitoesteróis, saponinas e limonoides; também os compostos nitrogenados (glucosinalatos) e os metabólitos fenólicos, incluindo os ácidos fenólicos, polifenóis e flavonoides (ANJO, 2004).

Dentre a classe dos carotenoides, encontra-se a luteína, que pertence à família das xantofilas e está sendo associada à diminuição do risco de desenvolver certas doenças, como doenças oculares, doenças cardiovasculares e câncer. Também se destaca como protetor da pele contra a exposição excessiva aos raios ultravioleta (ALTERNATIVE MEDICINE REVIEW, 2005; ALVES-RODRIGUES e SHAO, 2004; GAMA e SYLOS, 2007).

Dentre os flavonoides, encontram-se as catequinas, uma das seis classes dos flavonoides, que incluem galato de epigalocatequina (EGCG), epigalocatequina (EGC), epicatequina galato (ECG) e epicatequina (EC) (ASOLINI et al., 2006; CHO et al., 2007). Os flavonoides são reconhecidamente agentes antioxidantes capazes de inibir a oxidação de lipoproteínas de baixa densidade, além de estes reduzirem significativamente as tendências a doenças trombóticas (RAUHA et al., 2000).

No setor de bebidas, destaca-se o mercado crescente para sucos ou néctares mistos, compostos principalmente de frutas tropicais. Outra tendência é a adição de fitoquímicos (compostos bioativos) com alegações funcionais, visando à elaboração de uma bebida de frutas com efeitos benéficos à saúde. Porém, estas combinações geram produtos com comportamento reológico ainda não descrito na literatura, necessitando assim de estudos nesta área.

O comportamento reológico dos sucos é influenciado pela sua composição tanto quantitativa quanto qualitativa - como, exemplo, teor de pectina e de sólidos suspensos - e, por consequência, dependerá do tipo de fruta e dos tratamentos realizados no seu processo de elaboração (PELEGRINE et al., 2000). Deste modo, para se obter um produto de qualidade, as indústrias devem respeitar as características de cada fruta.

Segundo Vidal et al. (2004), a inexistência de dados reológicos sobre os sucos de frutas tropicais na literatura tem levado a indústria nacional a utilizar, no processo de fabricação destes sucos, condições semelhantes às aplicadas na produção do suco de laranja. Todavia, por terem propriedades diferentes, os resultados não atingem o mesmo nível de qualidade. A crescente necessidade e a procura dos parâmetros reológicos para os diversos fluidos manipulados nas indústrias de processamento estão ligadas também à grande importância econômica que estes fluidos e equipamentos de manipulação representam atualmente.

O conhecimento do comportamento reológico de alimentos fluidos é essencial para o projeto de equipamentos de processamento de alimentos, além de ser um parâmetro de grande importância para controle de qualidade, aceitabilidade dos consumidores e desenvolvimento de novos produtos, e na determinação da sua vida de prateleira (LEITE et al., 2004; RAMOS, 1997; RODRIGUES et al., 2003). Para compreender o comportamento de fluidos não newtonianos, que relacionam os dados de tensão de cisalhamento com a taxa de deformação, são utilizados alguns modelos matemáticos, como: Ostwald-de-Waele, Herschel-Bulkley e Casson, representados pelas Equações 1, 2 e 3, respectivamente (SILVA et al., 2005). Note-se que $\tau$ é a tensão de cisalhamento (Pa) e $\dot{\gamma}$ é a taxa de deformação $\left(s^{-1}\right)$, para todos os modelos.

$\tau=K \cdot \dot{\gamma}^{\mathrm{n}}$

$\mathrm{K}=$ índice de consistência $\left(\mathrm{Pa} \cdot \mathrm{s}^{\mathrm{n}}\right) ; \mathrm{n}=$ índice de comportamento do escoamento (adimensional).

$$
\tau=\tau_{0}+\mathrm{K}_{\mathrm{H}}(\dot{\gamma})^{\mathrm{n}_{\mathrm{H}}}
$$

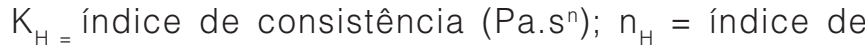
comportamento ao escoamento (adimensional); $\tau_{0=}$ tensão inicial $(\mathrm{Pa})$.

$\tau^{0,5}=\mathrm{K}_{\mathrm{oc}}+\mathrm{K}_{\mathrm{c}}(\dot{\gamma})^{0,5}$

$\mathrm{K}_{\mathrm{oc}}=$ tensão inicial $(\mathrm{Pa}) ; \mathrm{K}_{\mathrm{c}}=$ índice de consistência $\left(\mathrm{Pa} . \mathrm{S}^{0,5}\right)$.

Os modelos reológicos são usados para uma melhor descrição do comportamento reológico dos fluidos, permitindo relacionar as propriedades reológicas com grandezas práticas, como concentração, temperatura e índice de maturação (BRANCO, 1995).

Considerando-se que os alimentos fluidos durante o seu processamento são submetidos constantemente 
Propriedades reológicas de sucos mistos de manga, goiaba e acerola adicionados de fitoquímicos

FARAONI, A. S. et al.

a mudanças de temperatura, como, por exemplo, temperaturas de pasteurização e resfriamento, torna-se importante conhecer suas propriedades reológicas em função da temperatura.

O efeito da temperatura sobre a viscosidade aparente pode ser descrito mediante uma equação análoga à de Arrhenius:

$$
\eta_{\mathrm{a}}=\eta_{0} \exp \left(\frac{\mathrm{E}_{\mathrm{a}}}{\mathrm{RT}}\right)
$$

$\eta_{\mathrm{a}}=$ viscosidade aparente (Pa.s); $\eta_{0}=$ constante (Pa.s); $\mathrm{E}_{\mathrm{a}}=$ energia de ativação para escoamento viscoso (kJ. $\left.\mathrm{mol}^{-1}\right) ; \mathrm{R}=$ constante dos gases $\left(8,314 \cdot 10^{-3} \mathrm{~J} \cdot \mathrm{mol}^{-1} \cdot \mathrm{K}^{-1}\right)$; $\mathrm{T}=$ temperatura absoluta $(\mathrm{K})$.

A Equação de Arrhenius indica a tendência de diminuição da viscosidade aparente com o aumento da temperatura. De um modo geral, quanto maior a energia de ativação, maior será o efeito da temperatura sobre a viscosidade (RAO, 1986).

Para fluidos não newtonianos cujo comportamento reológico pode ser descrito pelo modelo de Ostwaldde-Waele, a viscosidade aparente é determinada pela seguinte equação:

$\eta_{\mathrm{a}}=\mathrm{K} \cdot \dot{\gamma}^{(\mathrm{n}-1)}$

O objetivo deste trabalho foi determinar as propriedades reológicas de suco misto de manga, goiaba e acerola adicionado de fitoquímicos em sete temperaturas, além de estudar o efeito deste fator sobre a viscosidade aparente.

\section{Material e métodos}

Para a elaboração dos sucos, foram utilizadas polpas pasteurizadas e congeladas de acerola e goiaba provenientes da Bela Ischia Ind. e Com. de Polpa e Fruta Congelada Ltda. e polpa de manga pasteurizada da variedade Ubá, comercializada pela Agrofruit Internacional do Brasil Ltda. Os fitoquímicos utilizados foram epigalocatequina galato e luteína, provenientes da empresa DSM Nutritional Products. Para sua conservação, foram utilizados benzoato de sódio (260 mg. $\mathrm{L}^{-1}$ ) e metabissulfito de sódio (40 mg. $\left.\mathrm{L}^{-1}\right)$. O teor de sólidos solúveis totais foi fixado em $11^{\circ}$ Brix e o teor da mistura de polpas, em 35\%.

Foram utilizados $13,65 \%$ de polpa de manga, $18,20 \%$ de polpa de goiaba e 3,15\% de polpa de acerola, sendo estas proporções escolhidas por meio de testes de aceitação, dentre dez tratamentos obtidos de um delineamento simplex de misturas (BARROS NETO et al., 1995), de acordo com a Tabela 1.

As polpas foram pesadas e homogeneizadas juntamente com água mineral e sacarose comercial, sendo adicionados os fitoquímicos luteína e epigalocatequina galato em quantidades apresentadas na Tabela 2.

A concentração da luteína foi determinada com base na faixa recomendada pela literatura (6-30 mg/dia) (ALTERNATIVE MEDICINE REVIEW, 2005; KRINSKY et al., 2003; KRINSKY e JOHNSON, 2005) e as concentrações da epigalocatequina galato, segundo recomendação dos fornecedores $(9,4-14,1 \mathrm{mg} / 100 \mathrm{~mL})$. Para cada formulação, foram feitas três repetições. A pasteurização dos sucos foi realizada a uma temperatura de $90{ }^{\circ} \mathrm{C} / 60$ s (MAlA et al., 2007) e, após o envase a quente em

Tabela 1. Delineamento simplex aumentado de dez tratamentos para as formulações das misturas de frutas.

\begin{tabular}{|c|c|c|c|c|c|c|}
\hline \multirow[t]{2}{*}{ Formulação } & \multicolumn{4}{|c|}{$\begin{array}{l}\text { Polpas } \\
\text { Goiaba }\end{array}$} & \multicolumn{2}{|c|}{ Acerola } \\
\hline & $\mathbf{R}$ & $\mathbf{N}$ & $\mathbf{R}$ & $\mathbf{N}$ & $\mathbf{R}$ & $\mathbf{N}$ \\
\hline 1 & 21,00 & 0,60 & 12,25 & 0,35 & 1,75 & 0,05 \\
\hline 2 & 12,25 & 0,35 & 21,00 & 0,60 & 1,75 & 0,05 \\
\hline 3 & 12,25 & 0,35 & 12,25 & 0,35 & 10,50 & 0,30 \\
\hline 4 & 16,80 & 0,48 & 16,80 & 0,48 & 1,75 & 0,05 \\
\hline 5 & 16,80 & 0,48 & 12,25 & 0,35 & 6,30 & 0,18 \\
\hline 6 & 12,25 & 0,35 & 16,80 & 0,48 & 6,30 & 0,18 \\
\hline 7 & 15,05 & 0,43 & 15,05 & 0,43 & 4,55 & 0,13 \\
\hline 8 & 18,20 & 0,52 & 13,65 & 0,39 & 3,15 & 0,09 \\
\hline 9 & 13,65 & 0,39 & 18,20 & 0,52 & 3,15 & 0,09 \\
\hline 10 & 13,65 & 0,39 & 13,65 & 0,39 & 7,70 & 0,22 \\
\hline
\end{tabular}

R: proporções reais (considerando-se a formulação como um todo); N: proporções normalizadas (considerando-se apenas o total das três polpas).

Tabela 2. Concentrações dos fitoquímicos, luteína e epigalocatequina galato (EGCG), nos sucos mistos.

\begin{tabular}{lcc}
\multicolumn{1}{c}{ Formulações } & \multicolumn{2}{c}{$\begin{array}{c}\text { Concentração dos fitoquímicos } \\
\text { EGCG (mg.L-1) }\end{array}$} \\
Suco Controle & - & - \\
Suco adicionado de luteína & 14 & - \\
Suco adicionado de EGCG & - & 125,02 \\
Suco adicionado de luteína/EGCG & 14 & 109,98 \\
\hline
\end{tabular}


Propriedades reológicas de sucos mistos de manga, goiaba e acerola adicionados de fitoquímicos FARAONI, A. S. et al.

garrafas de vidro com tampas plásticas rosqueáveis com capacidade para $318 \mathrm{~mL}$ e resfriamento em água corrente, os sucos foram armazenados à temperatura ambiente $\left(27 \pm 2{ }^{\circ} \mathrm{C}\right)$.

As leituras para determinação das medidas reológicas foram realizadas em um reômetro de cilindros concêntricos tipo Searle, marca Brookfield, modelo R/S Plus, acoplado a um banho-maria, marca Brookfield, modelo TC-502P, utilizando-se o sensor DG DIN para todas as amostras. As medidas reológicas foram realizadas às temperaturas de $10,20,30,40,50,60$ e $70^{\circ} \mathrm{C}$. O tempo de corrida, para cada ensaio, foi programado para dois minutos (corrida ascendente), com taxa de deformação variando de 0 a $800 \mathrm{~s}^{-1}$, obtendo-se 24 pontos de taxa de deformação versus tensão de cisalhamento. As leituras foram realizadas em duplicata para cada temperatura.

A viscosidade aparente foi calculada utilizando-se a Equação 5 e com base em uma taxa de deformação de $100 \mathrm{~s}^{-1}$, valor característico de diversos processos aplicados a alimentos (SATO e CUNHA, 2007).
Os valores experimentais de tensão de cisalhamento e da taxa de deformação foram ajustados pelos modelos de Ostwald-de-Waele (Lei da Potência), Casson e Herschel-Bulkley.

Os dados experimentais foram processados utilizando-se o Programa SAS ${ }^{\circledR}$ licenciado pela UFV. Para o ajuste dos modelos, foram determinados dois parâmetros estatísticos: coeficiente de determinação $\left(R^{2}\right)$ e quadrado médio do erro (QME).

\section{Resultados e discussão}

\subsection{Comportamento reológico}

Nas Tabelas 3 a 5, estão indicados os parâmetros dos três modelos utilizados para os ajustes dos dados experimentais e os respectivos índices de ajuste.

Observou-se que os três modelos apresentaram altos coeficientes de determinação $\left(R^{2} \geq 0,978\right)$, indicando que qualquer um destes pode ser utilizado para descrever o comportamento reológico dos sucos em estudo

Tabela 3. Parâmetros do modelo de Ostwald-de-Waele para as diferentes formulações dos sucos.

\begin{tabular}{|c|c|c|c|c|}
\hline \multicolumn{5}{|c|}{ Suco controle } \\
\hline $\mathrm{T}\left({ }^{\circ} \mathbf{C}\right)$ & K(Pa.s $\left.\mathbf{s}^{n}\right)$ & $n$ & $\mathbf{R}^{2}$ & QME \\
\hline 10 & $0,100 \pm 0,005$ & $0,637 \pm 0,009$ & 0,998 & 0,0076 \\
\hline 20 & $0,070 \pm 0,004$ & $0,660 \pm 0,010$ & 0,997 & 0,0063 \\
\hline 30 & $0,029 \pm 0,001$ & $0,754 \pm 0,006$ & 0,999 & 0,0013 \\
\hline 40 & $0,047 \pm 0,003$ & $0,664 \pm 0,009$ & 0,998 & 0,0024 \\
\hline 50 & $0,036 \pm 0,003$ & $0,665 \pm 0,013$ & 0,995 & 0,0029 \\
\hline 60 & $0,045 \pm 0,005$ & $0,613 \pm 0,016$ & 0,991 & 0,0029 \\
\hline 70 & $0,066 \pm 0,006$ & $0,551 \pm 0,015$ & 0,989 & 0,0040 \\
\hline \multicolumn{5}{|c|}{ Suco adicionado de luteína } \\
\hline 10 & $0,074 \pm 0,003$ & $0,681 \pm 0,007$ & 0,999 & 0,0046 \\
\hline 20 & $0,076 \pm 0,004$ & $0,648 \pm 0,008$ & 0,998 & 0,0042 \\
\hline 30 & $0,083 \pm 0,009$ & $0,612 \pm 0,017$ & 0,990 & 0,0153 \\
\hline 40 & $0,045 \pm 0,003$ & $0,663 \pm 0,012$ & 0,996 & 0,0039 \\
\hline 50 & $0,058 \pm 0,067$ & $0,606 \pm 0,018$ & 0,987 & 0,0083 \\
\hline 60 & $0,056 \pm 0,004$ & $0,589 \pm 0,012$ & 0,994 & 0,0029 \\
\hline 70 & $0,078 \pm 0,007$ & $0,525 \pm 0,014$ & 0,990 & 0,0035 \\
\hline \multicolumn{5}{|c|}{ Suco adicionado de EGCG } \\
\hline 10 & $0,068 \pm 0,005$ & $0,692 \pm 0,011$ & 0,997 & 0,0104 \\
\hline 20 & $0,067 \pm 0,005$ & $0,666 \pm 0,013$ & 0,995 & 0,0102 \\
\hline 30 & $0,085 \pm 0,006$ & $0,607 \pm 0,012$ & 0,995 & 0,0072 \\
\hline 40 & $0,064 \pm 0,009$ & $0,612 \pm 0,022$ & 0,982 & 0,0156 \\
\hline 50 & $0,060 \pm 0,007$ & $0,598 \pm 0,019$ & 0,986 & 0,0088 \\
\hline 60 & $0,043 \pm 0,004$ & $0,639 \pm 0,014$ & 0,994 & 0,0038 \\
\hline 70 & $0,073 \pm 0,010$ & $0,541 \pm 0,021$ & 0,978 & 0,0086 \\
\hline \multicolumn{5}{|c|}{ Suco adicionado de luteína e EGCG } \\
\hline 10 & $0,061 \pm 0,002$ & $0,705 \pm 0,006$ & 0,999 & 0,0033 \\
\hline 20 & $0,056 \pm 0,004$ & $0,684 \pm 0,012$ & 0,996 & 0,0075 \\
\hline 30 & $0,070 \pm 0,007$ & $0,624 \pm 0,017$ & 0,990 & 0,0124 \\
\hline 40 & $0,056 \pm 0,004$ & $0,632 \pm 0,013$ & 0,994 & 0,0050 \\
\hline 50 & $0,038 \pm 0,003$ & $0,666 \pm 0,011$ & 0,996 & 0,0026 \\
\hline 60 & $0,058 \pm 0,006$ & $0,587 \pm 0,017$ & 0,989 & 0,0055 \\
\hline 70 & $0,055 \pm 0,005$ & $0,573 \pm 0,015$ & 0,990 & 0,0036 \\
\hline
\end{tabular}


Propriedades reológicas de sucos mistos de manga, goiaba e acerola adicionados de fitoquímicos

FARAONI, A. S. et al.

Tabela 4. Parâmetros do modelo de Herschel-Bulkley para as diferentes formulações dos sucos.

\begin{tabular}{|c|c|c|c|c|c|}
\hline \multicolumn{6}{|c|}{ Suco controle } \\
\hline $\mathrm{T}\left({ }^{\circ} \mathbf{C}\right)$ & $\mathrm{K}_{\mathrm{H}}\left(\mathrm{Pa} . \mathbf{s}^{\mathrm{n}}\right)$ & $\mathbf{n}_{\mathrm{H}}$ & $\tau_{0}(\mathrm{~Pa})$ & $\mathbf{R}^{2}$ & QME \\
\hline 10 & $0,049 \pm 0,007$ & $0,735 \pm 0,020$ & $0,511 \pm 0,085$ & 0,999 & 0,0033 \\
\hline 20 & $0,035 \pm 0,006$ & $0,754 \pm 0,025$ & $0,382 \pm 0,086$ & 0,998 & 0,0036 \\
\hline 30 & $0,018 \pm 0,001$ & $0,816 \pm 0,012$ & $0,173 \pm 0,028$ & 1,000 & 0,0005 \\
\hline 40 & $0,022 \pm 0,002$ & $0,769 \pm 0,016$ & $0,289 \pm 0,037$ & 0,998 & 0,0024 \\
\hline 50 & $0,012 \pm 0,002$ & $0,821 \pm 0,023$ & $0,312 \pm 0,037$ & 0,999 & 0,0008 \\
\hline 60 & $0,007 \pm 0,001$ & $0,863 \pm 0,020$ & $0,432 \pm 0,024$ & 0,999 & 0,0004 \\
\hline 70 & $0,011 \pm 0,002$ & $0,795 \pm 0,031$ & $0,456 \pm 0,040$ & 0,998 & 0,0009 \\
\hline \multicolumn{6}{|c|}{ Suco adicionado de luteína } \\
\hline 10 & $0,042 \pm 0,005$ & $0,756 \pm 0,015$ & $0,367 \pm 0,064$ & 0,999 & 0,0020 \\
\hline 20 & $0,037 \pm 0,004$ & $0,747 \pm 0,014$ & $0,411 \pm 0,050$ & 0,999 & 0,0012 \\
\hline 30 & $0,013 \pm 0,002$ & $0,870 \pm 0,020$ & $0,806 \pm 0,045$ & 0,999 & 0,0014 \\
\hline 40 & $0,018 \pm 0,004$ & $0,790 \pm 0,028$ & $0,323 \pm 0,059$ & 0,998 & 0,0019 \\
\hline 50 & $0,008 \pm 0,002$ & $0,875 \pm 0,031$ & $0,561 \pm 0,045$ & 0,998 & 0,0015 \\
\hline 60 & $0,015 \pm 0,003$ & $0,765 \pm 0,025$ & $0,364 \pm 0,039$ & 0,998 & 0,0008 \\
\hline 70 & $0,013 \pm 0,002$ & $0,764 \pm 0,023$ & $0,472 \pm 0,031$ & 0,999 & 0,0005 \\
\hline \multicolumn{6}{|c|}{ Suco adicionado de EGCG } \\
\hline 10 & $0,028 \pm 0,004$ & $0,816 \pm 0,020$ & $0,556 \pm 0,072$ & 0,999 & 0,0031 \\
\hline 20 & $0,025 \pm 0,005$ & $0,802 \pm 0,028$ & $0,523 \pm 0,009$ & 0,998 & 0,0044 \\
\hline 30 & $0,033 \pm 0,007$ & $0,734 \pm 0,030$ & $0,471 \pm 0,090$ & 0,997 & 0,0037 \\
\hline 40 & $0,006 \pm 0,001$ & $0,945 \pm 0,032$ & $0,745 \pm 0,047$ & 0,998 & 0,0020 \\
\hline 50 & $0,009 \pm 0,002$ & $0,863 \pm 0,040$ & $0,556 \pm 0,058$ & 0,996 & 0,0024 \\
\hline 60 & $0,010 \pm 0,001$ & $0,842 \pm 0,015$ & $0,403 \pm 0,023$ & 0,999 & 0,0003 \\
\hline 70 & $0,005 \pm 0,001$ & $0,908 \pm 0,043$ & $0,623 \pm 0,045$ & 0,996 & 0,0016 \\
\hline \multicolumn{6}{|c|}{ Suco adicionado de luteína e EGCG } \\
\hline 10 & $0,036 \pm 0,003$ & $0,779 \pm 0,010$ & $0,333 \pm 0,040$ & 1,000 & 0,0009 \\
\hline 20 & $0,020 \pm 0,003$ & $0,825 \pm 0,020$ & $0,488 \pm 0,056$ & 0,999 & 0,0020 \\
\hline 30 & $0,012 \pm 0,002$ & $0,863 \pm 0,026$ & $0,690 \pm 0,053$ & 0,998 & 0,0020 \\
\hline 40 & $0,015 \pm 0,002$ & $0,816 \pm 0,018$ & $0,465 \pm 0,035$ & 0,999 & 0,0008 \\
\hline 50 & $0,013 \pm 0,001$ & $0,817 \pm 0,013$ & $0,323 \pm 0,023$ & 1,000 & 0,0003 \\
\hline 60 & $0,013 \pm 0,004$ & $0,792 \pm 0,045$ & $0,423 \pm 0,068$ & 0,995 & 0,0026 \\
\hline 70 & $0,009 \pm 0,002$ & $0,815 \pm 0,026$ & $0,418 \pm 0,032$ & 0,998 & 0,0006 \\
\hline
\end{tabular}

(Tabelas 3 a 5). O modelo de Ostwald-de-Waele seria o mais indicado por ser simples e de ampla aplicação tecnológica (BRANCO e GASPARETO, 2003), além de ser bastante utilizado para descrever o comportamento reológico de sucos e néctares.

Analisando-se os modelos de Ostwald-de-Waele e Herschel-Bulkley, verificou-se que os valores de $n$ e $\mathrm{n}_{\mathrm{H}}$ (índices de comportamento do escoamento) foram menores do que a unidade, indicando comportamento pseudoplástico do fluido; note-se que quanto mais afastado da unidade, maior a pseudoplasticidade do produto. Não foi observada uma influência marcante do aumento da temperatura nos valores de $n$ e $n_{H}$, uma vez que se mantiveram praticamente constantes.

Os valores de $n$ e $n_{H}$ encontrados neste trabalho estão próximos dos valores apresentados por Vandresen et al. (2009) ao estudarem o efeito da temperatura no comportamento reológico de suco de cenoura pasteurizado.

Verificou-se também a redução nas viscosidades aparentes com o aumento da temperatura, confirmando a pseudoplasticidade dos sucos $(n<1)$. Uma redução mínima da viscosidade aparente, tendendo-se à estabilidade, foi observada nas temperaturas de $60{ }^{\circ} \mathrm{C}$ e $70^{\circ} \mathrm{C}$ para os sucos controle e adicionados de luteína, e a partir da temperatura de $50^{\circ} \mathrm{C}$ para os sucos adicionados de epigalocatequina galato e luteína/epigalocatequina galato.

Nos sucos, os índices de consistência $\mathrm{K}, \mathrm{K}_{\mathrm{c}} \mathrm{e}$ $\mathrm{K}_{\mathrm{H}}$, de um modo geral, decresceram com o aumento de temperatura. Este comportamento também foi observado por Silva et al. (2005), ao analisarem o efeito da temperatura nos parâmetros reológicos de suco de acerola, e por Cabral et al. (2002), ao estudarem 
Propriedades reológicas de sucos mistos de manga, goiaba e acerola adicionados de fitoquímicos

FARAONI, A. S. et al.

Tabela 5. Parâmetros do modelo de Casson para as diferentes formulações dos sucos.

\begin{tabular}{|c|c|c|c|c|}
\hline \multicolumn{5}{|c|}{ Suco controle } \\
\hline $\mathrm{T}\left({ }^{\circ} \mathbf{C}\right)$ & $\mathrm{K}_{\mathrm{oc}}(\mathrm{Pa})$ & $\mathrm{K}_{\mathrm{c}}\left(\mathrm{Pa} . \mathrm{s}^{0,5}\right)$ & $\mathbf{R}^{2}$ & QME \\
\hline 10 & $0,690 \pm 0,025$ & $0,071 \pm 0,001$ & 0,996 & 0,0157 \\
\hline 20 & $0,581 \pm 0,023$ & $0,065 \pm 0,001$ & 0,996 & 0,0100 \\
\hline 30 & $0,367 \pm 0,012$ & $0,062 \pm 0,001$ & 0,999 & 0,0019 \\
\hline 40 & $0,479 \pm 0,017$ & $0,054 \pm 0,001$ & 0,997 & 0,0037 \\
\hline 50 & $0,423 \pm 0,015$ & $0,048 \pm 0,001$ & 0,997 & 0,0023 \\
\hline 60 & $0,463 \pm 0,017$ & $0,043 \pm 0,001$ & 0,995 & 0,0029 \\
\hline 70 & $0,526 \pm 0,021$ & $0,039 \pm 0,001$ & 0,990 & 0,0047 \\
\hline \multicolumn{5}{|c|}{ Suco adicionado de luteína } \\
\hline 10 & $0,599 \pm 0,021$ & $0,073 \pm 0,001$ & 0,997 & 0,0098 \\
\hline 20 & $0,605 \pm 0,021$ & $0,065 \pm 0,001$ & 0,997 & 0,0091 \\
\hline 30 & $0,626 \pm 0,024$ & $0,058 \pm 0,001$ & 0,994 & 0,010 \\
\hline 40 & $0,469 \pm 0,018$ & $0,053 \pm 0,001$ & 0,996 & 0,004 \\
\hline 50 & $0,520 \pm 0,022$ & $0,047 \pm 0,001$ & 0,993 & 0,0058 \\
\hline 60 & $0,500 \pm 0,019$ & $0,043 \pm 0,001$ & 0,994 & 0,0038 \\
\hline 70 & $0,556 \pm 0,022$ & $0,038 \pm 0,001$ & 0,989 & 0,0054 \\
\hline \multicolumn{5}{|c|}{ Suco adicionado de EGCG } \\
\hline 10 & $0,582 \pm 0,021$ & $0,074 \pm 0,001$ & 0,998 & 0,0094 \\
\hline 20 & $0,576 \pm 0,023$ & $0,065 \pm 0,001$ & 0,996 & 0,0102 \\
\hline 30 & $0,627 \pm 0,026$ & $0,057 \pm 0,001$ & 0,993 & 0,0121 \\
\hline 40 & $0,555 \pm 0,026$ & $0,051 \pm 0,001$ & 0,992 & 0,0093 \\
\hline 50 & $0,528 \pm 0,024$ & $0,046 \pm 0,001$ & 0,991 & 0,0068 \\
\hline 60 & $0,459 \pm 0,015$ & $0,047 \pm 0,001$ & 0,996 & 0,0026 \\
\hline 70 & $0,549 \pm 0,025$ & $0,040 \pm 0,001$ & 0,986 & 0,0070 \\
\hline \multicolumn{5}{|c|}{ Suco adicionado de luteína e EGCG } \\
\hline 10 & $0,546 \pm 0,018$ & $0,074 \pm 0,001$ & 0,998 & 0,0066 \\
\hline 20 & $0,527 \pm 0,019$ & $0,064 \pm 0,001$ & 0,997 & 0,0060 \\
\hline 30 & $0,582 \pm 0,023$ & $0,056 \pm 0,001$ & 0,995 & 0,0086 \\
\hline 40 & $0,518 \pm 0,018$ & $0,052 \pm 0,001$ & 0,996 & 0,0042 \\
\hline 50 & $0,435 \pm 0,014$ & $0,049 \pm 0,001$ & 0,998 & 0,0021 \\
\hline 60 & $0,511 \pm 0,023$ & $0,043 \pm 0,001$ & 0,991 & 0,0058 \\
\hline 70 & $0,490 \pm 0,019$ & $0,039 \pm 0,001$ & 0,992 & 0,0035 \\
\hline
\end{tabular}

o comportamento reológico da polpa de cupuaçu peneirada.

Com relação à tensão inicial $\left(\tau_{0}\right)$, não foi possível observar um comportamento frente ao aumento da temperatura. No entanto, observam-se valores muito baixos ( $\tau_{0}$ médio de 0,400 Pa) para os modelos de Herschel-Bulkley e Casson, podendo ser uma indicação de que o modelo de Ostwald-de-Waele é o mais adequado para descrever o comportamento reológico destes produtos.

\subsection{Efeito da temperatura}

Os valores de $\eta_{0}$ (constante) e $E_{a}$ (energia de ativação) foram obtidos por meio da utilização da Equação de Arrhenius (Equação 4), mediante uma regressão linear. Os valores são mostrados na Tabela 6 , juntamente com seus respectivos índices de ajustes estatísticos

O valor da energia de ativação para o suco controle foi de $11,66 \mathrm{~kJ} \cdot \mathrm{mol}^{-1}$, o que indica que a temperatura exerceu um maior efeito na viscosidade aparente deste produto.

Na Figura 1, é mostrada a variação da viscosidade aparente com o inverso da temperatura, em que estão plotados os pontos experimentais observados e calculados pela equação de Arrhenius.

Na Figura 1, observa-se uma tendência geral de diminuição da viscosidade aparente com o aumento da temperatura, como indica a equação do tipo Arrhenius. De acordo com Barbosa-Cánovas et al. (1993), a viscosidade de um fluido está relacionada com forças intermoleculares que impedem o movimento das moléculas e estas forças dependem da extensão dos espaços intermoleculares, 
Propriedades reológicas de sucos mistos de manga, goiaba e acerola adicionados de fitoquímicos FARAONI, A. S. et al.

Tabela 6. Parâmetros estimados pelo ajuste do modelo de Arrhenius com base na viscosidade aparente $\left(\dot{\gamma}=100 \mathrm{~s}^{-1}\right)$.

\begin{tabular}{|c|c|c|c|c|}
\hline \multirow{2}{*}{ Formulações } & \multicolumn{4}{|c|}{ Parâmetros estimados } \\
\hline & $\eta_{0}$ (Pa.s) & $\mathrm{E}_{\mathrm{a}}\left(\mathrm{kJ} \cdot \mathrm{mol}^{-1}\right)$ & $\mathbf{R}^{2}$ & QME \\
\hline Suco controle & $1,14876 \times 10^{-4}$ & 11,66314 & 0,770 & 0,0271 \\
\hline Suco adicionado de luteína & $2,11601 \times 10^{-4}$ & 10,31622 & 0,886 & 0,0096 \\
\hline Suco adicionado de epigalocatequina galato (EGCG) & $2,52646 \times 10^{-4}$ & 9,86085 & 0,919 & 0,0060 \\
\hline Suco adicionado de luteína + EGCG & $2,40228 \times 10^{-4}$ & 9,79816 & 0,939 & 0,0044 \\
\hline
\end{tabular}

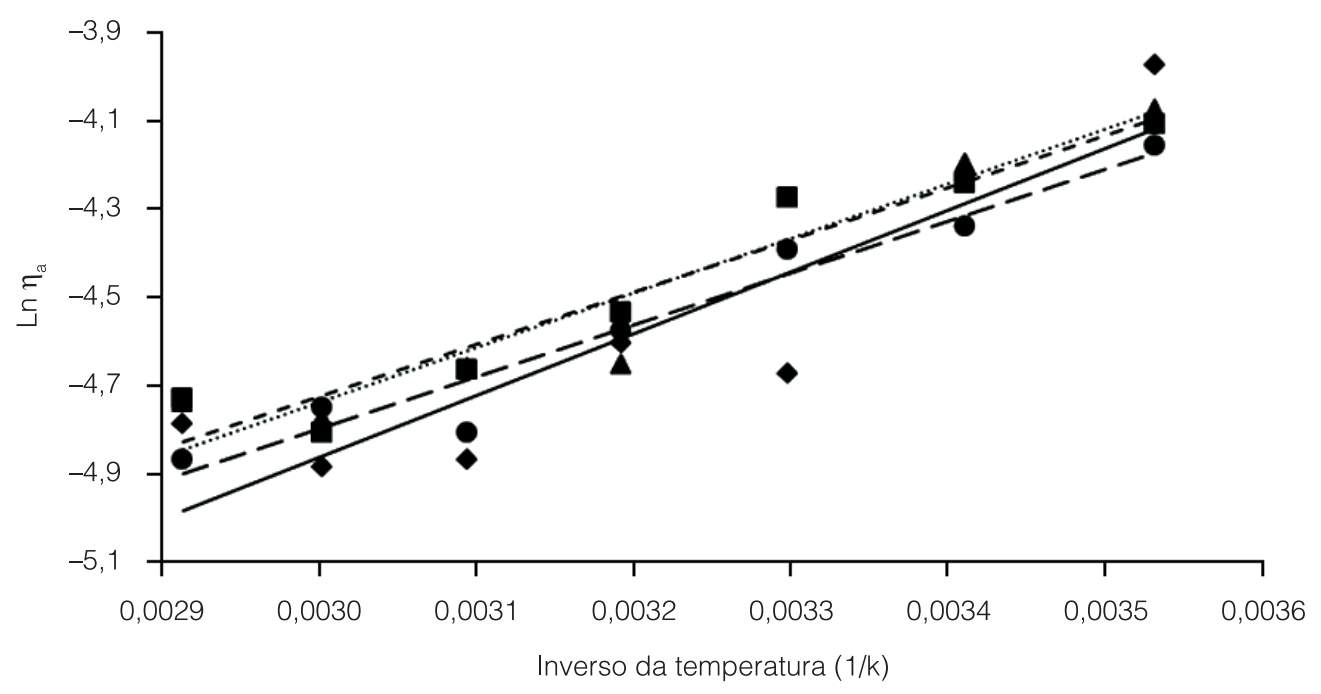
- Suco controle obs
$\Delta$ Suco adicionado de luteína obs
- Suco adicionado de EGCG obs
Suco controle est
............. Suco adicionado de luteína est
- Suco adicionado de luteína/EGCG obs
- - - - Suco adicionado de EGCG est
- - - Suco adicionado de luteína/EGCG est

Figura 1. Efeito da temperatura sobre a viscosidade aparente dos sucos, segundo a Equação de Arrhenius.

que determinam seu volume livre, e são influenciadas por mudanças de temperatura e pressão.

\section{Conclusões}

Os sucos mistos podem ser descritos pelos três modelos analisados, sendo que o modelo de Ostwald-deWaele foi o indicado por ser simples e de ampla aplicação tecnológica.

Os valores dos índices de comportamento ao escoamento foram inferiores à unidade, caracterizando os sucos como um fluido não newtoniano, com características pseudoplásticas.

Não foi observada uma influência marcante do aumento da temperatura nos valores de índice de comportamento ao escoamento. No entanto, os valores de índice de consistência apresentaram tendência decrescente com o aumento de temperatura.
Observou-se que o modelo de Arrhenius explicou satisfatoriamente a variação da viscosidade aparente com a temperatura.

\section{Agradecimentos}

Os autores agradecem à FAPEMIG pelo auxílio financeiro.

\section{Referências}

ANJO, D. F. C. Alimentos funcionais em angiologia e cirurgia vascular. Jornal Vascular Brasileiro, Porto Alegre, v. 3, n. 2, p. 145-154, 2004.

ALternative MEDICINE REVIEW. Lutein and Zeaxanthin - Monograph. Nenhum autor listado. Alternative Medicine Review, Napa, v. 10, n. 2, p. 128-135, 2005.

ALVES-RODRIGUES, A.; SHAO, A. The science behind lutein. Toxicology Letters, Amsterdam, v. 150, n. 1, p. 57-83, 2004. http://dx.doi.org/10.1016/j.toxlet.2003.10.031 
Propriedades reológicas de sucos mistos de manga, goiaba e acerola adicionados de fitoquímicos FARAONI, A. S. et al.

ASOLINI, F. C.; TEDESCO, A. M.; CARPES, S. T.; FERRAZ, C.; ALENCAR, S. M. Atividade antioxidante e antibacteriana dos compostos fenólicos dos extratos de plantas usadas como chás. Brazilian Journal of Food Technology, Campinas, v. 9 , n. 3, p. 209-215, 2006.

BARBOSA-CÁNOVAS, G. V.; IRBAZ, A.; PELEG, M. Propriedades reológicas de alimentos fluidos: Revision. Alimentaria, Madrid, v. 241, p. 36-89, 1993.

BARROS NETO, B.; SCARMINIO, I. S.; BRUMS, R. E. Planejamento e Otimização de Experimentos. 2. ed. Campinas: Editora da UNICAMP, 1995. 299 p.

BRANCO, I. G. Suco de Laranja Concentrado: Comportamento Reológico a Baixas Temperaturas. 1995. 91 f. Dissertação (Mestrado em Engenharia de Alimentos)-Faculdade de Engenharia de Alimentos, Universidade Estadual de Campinas, Campinas, 1995.

BRANCO, I. G.; GASPARETTO, C. A. Aplicação da metodologia de superfície de resposta para o estudo do efeito da temperatura sobre o comportamento reológico de misturas ternárias de polpa de manga e sucos de laranja e cenoura. Ciência e Tecnologia de Alimentos, Campinas, v. 23, p. 166-171, 2003. Supplemento. http://dx.doi.org/10.1590/S0101-20612003000400031

CABRAL, M. F. P.; QUEIROZ, A. J. M.; FIGUEIRÊDO, R. M. F. Comportamento reológico da polpa de cupuaçu (theobroma grandiflorum schum.) peneirada. Revista Brasileira de Produtos Agroindustriais, Campina Grande, v. 4, n. 1, p. 37-40, 2002.

CHO, K-N.; SUKHTHANKAR, M.; LEE, S-H.; YOON, J-H.; BAEK, S. J. Green tea catechin (-)-epicatechin gallate induces tumour suppressor protein ATF3 via EGR-1 activation. European Journal of Cancer, Oxford, v. 43, n. 16, p. 2404-2412, 2007. http://dx.doi.org/10.1016/j.ejca.2007.07.020

GAMA, J. J. T.; SYLOS, C. M. Effecft of thermal pasteurization and concentration on carotenoid composition of Brazilian Valencia orange juice. Food Chemistry, Barking, v. 100, n. 4, p. 1686-1690, 2007. http://dx.doi.org/10.1016/j. foodchem.2005.01.062

GOMES, F. S. Carotenóides: uma possível proteção contra o desenvolvimento de câncer. Revista de Nutrição, Campinas, v. 20, n. 5, p. 537-548, 2007. http://dx.doi.org/10.1590/S141552732007000500009

KRINSKY, N. I.; LANDRUM, J. T.; BONE, R. A. Biologic mechanisms of the protective role of lutein and zeaxanthin in the eye. Annual Review Nutrition, Boston, v. 23, p. 171-201, 2003. http://dx.doi.org/10.1146/annurev.nutr.23.011702.073307

KRINSKY, N. I.; JOHNSON E. J. Carotenoid Actions and their Relation to Health and Disease. Molecular Aspects of Medicine, Amsterdam, v. 26, n. 6, p. 459-516, 2005. http://dx. doi. org/10.1016/j.mam.2005.10.001

LEITE, J. T. C.; PARK, K. J.; RAMALHO, J. R. P.; FURLAN, D. M. Caracterização reológica das diferentes fases de extrato de inulina de raízes de chicória, obtidas por abaixamento de temperatura. Engenharia Agrícola, Jaboticabal, v. 24, n. 1, p. 202-210, 2004. http://dx.doi.org/10.1590/S010069162004000100023

MAIA, G. A.; SOUZA, P. H. M.; SANTOS, G. M.; SILVA, D. S.; FERNANDES, A. G.; PRADO, G.M. Efeito do processamento sobre componentes do suco de acerola. Ciência e Tecnologia de Alimentos, Campinas, v. 27, n. 1, p. 130-134, 2007. http:// dx.doi.org/10.1590/S0101-20612007000100023

PELEGRINE, D. H.; VIDAL, J. R. M. B.; GASPARETTO, C. A. Estudo da viscosidade aparente das polpas de manga (Keitt) e abacaxi (Pérola). Ciência e Tecnologia de Alimentos, Campinas, v. 20, n. 1, p. 128-131, 2000. http://dx.doi. org/10.1590/S0101-20612000000100024

RAMOS, A. M. Caracterización Reológica y Transmisión de Calor em Derivados de Frutas en el Interior de Tanques Agitados. 1997. 76 f. Tese (Doutorado em Ciência e Tecnologia de Alimentos)-Escola Técnica Superior D’Enginyeria Agrária de Lleida, Universitat de Lleida, Lleida, 1997.

RAO, M. A. Rheological properties of fluid foods. In: RAO, M. A.; RIZVI, S. S. H. Engineering Properties of Foods. New York: marcel dekker, 1986. cap. 5, p. 1-47.

RAUHA, J. P. et al. Antimicrobial effects of Finnish plant extracts containing flavonoids and other phenolic compounds. International Journal of Food Microbiology, Amsterdam, v. 56, n. 1, p. 3-12, 2000. http://dx.doi.org/10.1016/S01681605(00)00218-X

RODRIGUES, R. S.; GOZZO, Â. M.; MORETTI, R. H. Comportamento reológico de extratos de grãos, Farinha integral e isolado protéico de soja. Boletim do CEPPA, Curitiba, v. 21, n. 2, p. 367-378, 2003.

SATO, A. C. K.; CUNHA, R. L. Influência da temperatura no comportamento reológico da polpa de jabuticaba. Ciência e Tecnologia de Alimentos, Campinas, v. 27 , n. 4, p. 890-896, 2007. http://dx.doi.org/10.1590/S010120612007000400033

Silva, F. C.; Guimaraes, D. H. P.; GASPARETTO, C. A. Reologia do suco de acerola: efeitos da concentração e temperatura. Ciência e Tecnologia de Alimentos, Campinas, v. 25 , n. 1, p. 121-126, 2005. http://dx.doi.org/10.1590/S010120612005000100020

VANDRESEN, S.; QUADRI, M. G. N.; DE SOUZA, J. A. R.; HOTZA, D. Temperature effect on the rheological behavior of carrot juices. Journal of Food Engineering, Amsterdam, v. 92, n. 3, p. 269-274, 2009. http://dx.doi.org/10.1016/j. jfoodeng.2008.11.010

VIDAL, J. R. M. B.; PELEGRINE, D. H.; GASPARETTO, C. A. Efeito da temperatura no comportamento reológico da polpa de manga (mangífera indica L-keitt). Ciência e Tecnologia de Alimentos, Campinas, v. 24, n. 1, p. 39-42, 2004. http://dx.doi. org/10.1590/S0101-20612004000100008 